Acta Hispanica (2020) Supplementum II: 409-421

\title{
TENDENCIAS ACTUALES Y NUEVOS DESAFÍOS DE LOS MIGRANTES EN CHILE
}

\author{
GABRIELLA THOMAZY \\ Universidad Nacional de Servicio Público, Budapest
}

\begin{abstract}
Resumen: Las noticias internacionales, muchas veces, tratan de la migración hacia Europa o hacia los EE. UU. Muy pocas veces escuchamos de la migración dentro de América Latina, siendo Chile uno de los países elegidos dentro de la región entre la gente que quiere buscar un mejor futuro; no solo los grupos más vulnerables se movilizan hacia Chile, sino que, una parte de estas migraciones son protagonizadas por sujetos de estratos medios y altos, muchos con estudios técnicos o superiores. Chile no está preparado para el explosivo aumento que ha tenido la migración en los últimos años, legislaciones antiguas y cambios pequeños e inefectivos tratan de aliviar el colapso del sistema. El presente estudio tiene como objetivo principal, constatar cuáles son las tendencias actuales y los nuevos desafíos de los migrantes, sugiriendo importantes cambios, además de tratar los problemas de los migrantes en un entorno donde la religión e idioma generalmente es igual o similar. Las estadísticas disponibles, la legislación chilena u otros datos permiten analizar las tendencias y en base a eso los nuevos desafíos. ¿Realmente es más fácil buscar un nuevo futuro dentro de la región? ¿Chile podría facilitar la integración laboral de los migrantes? Palabras claves: migración, América Latina, Chile, estadísticas, legislación.
\end{abstract}

\begin{abstract}
International news often speaks about migration to Europe or to US. Very rarely do we hear about migration within Latin America. Chile is one of the countries chosen within the region among the people who want to find a better future and not only the most vulnerable groups are moving to Chile, but a part of these migrations are carried out by subjects of middle and upper strata, many with technical studies or higher. Chile is not prepared for this huge increase of migration in recent years. Old laws and small and ineffective changes try to prevent the collapse of the system. The main objective of this study is to verify what the current trends and new challenges of migrants are, suggest important changes, and deal with the problems of migrants in an environment where religion and language are generally the same or similar. Available statistics, Chilean legislation or other data allow to analyze trends and based on those the new challenges. Is it easier to find a new future within the region? Could Chile facilitate the labor integration of migrants?

Keywords: Migration, Latin America, Chile, Statistics, Legislation.
\end{abstract}


Tendencias actuales y nuevos desafíos de los migrantes en Chile

\section{Introducción}

Chile es un país bastante desconocido en Europa y muy pocas veces está en las noticias internacionales, generalmente se puede escuchar de este país lejano después de un terremoto u otro tipo de desastre natural.

Chile actualmente es un país atractivo para los migrantes, inversionistas y/u otras personas que quieren proyectarse en el futuro. Los datos económicos más importantes, como el PIB Anual: US\$298.172 M, PIB per cápita: US\$ 16.245, el crecimiento del PIB (anual): 4\% (FMI, 2018), la inflación: 2,6\%, la tasa de desempleo: 6,8\% (INE, 2018) ${ }^{1}$-son uno de los mejores indicadores en la región. Demográficamente es un país pequeño dentro de América Sur, con una población de 18.355.000 (INE, 2018).

Es importante mencionar que Chile permanece como el segundo país menos corrupto a nivel latinoamericano, después de Uruguay según el Índice de Percepción de la Corrupción, elaborado por la ONG Transparencia Internacional (2018), en el ranking mundial ocupa el No27 (A modo de ejemplo, Francia No21, España No41 y Hungría No64). Además, Chile es uno de los países más seguros en América Latina (Insight Crime, 2018).

Muchos migrantes buscan un mejor futuro en Chile y no solamente los grupos más vulnerables se movilizan hacia ese país, sino que también las personas calificadas con estudios, quienes forman parte de la clase media-alta. El presente estudio tiene como objetivo principal revisar las tendencias actuales y los nuevos desafíos de los migrantes. Analizando los datos disponibles, que permiten inferir posibles tendencias y en base de eso los nuevos desafíos. El presente estudio se realizó recopilando datos disponibles, principalmente del Departamento de Extranjería y Migración (DEM), además, listas públicas de la revalidación de títulos otorgados por la Universidad de Chile, revisando los datos desde 2005 hasta 2018. Además, entrevistas personales, la legislación laboral, incluyendo la ley de migración vigente, las políticas de migración y las propuestas de los cambios migratorios ayudaron a analizar la situación actual. Con este estudio el enfoque es buscar respuestas a las siguientes preguntas:

¿Realmente es más fácil buscar un nuevo futuro dentro de la región? ¿Chile podría facilitar la integración laboral de los migrantes?

\section{Datos estadísticos de los extranjeros}

Durante los últimos años, Chile ha experimentado un fenómeno migratorio muy significativo. Según una estimación realizada en conjunto, por el Instituto Nacional de Estadísticas (INE) y el DEM, en el Censo 2002, 195.320 personas extranjeras fueron censadas, mientras entre 2014 y diciembre del 2018 entraron al país cerca de 835.000 migrantes.

${ }^{1}$ INE - Instituto Nacional de Estadísticas, institución pública chilena. 


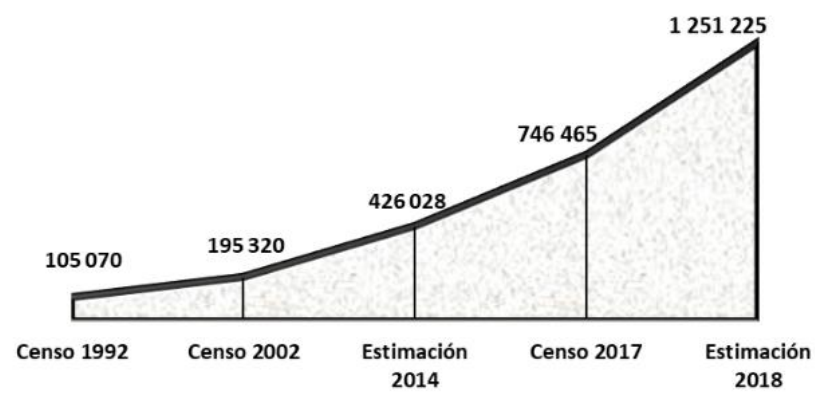

SEQ Gráfico \* ARABIC 1. Gráfico: Extranjeros en Chile (Fuente: INE, DEM)

El número de personas extranjeras residentes habituales en Chile llegó a unos 1.251.225 al 31 de diciembre de 2018, de las cuales 646.128 corresponderían a hombres y 605.097, a mujeres. (INE), es decir, las personas extranjeras residentes en Chile habrían alcanzado el 6,6\% de la población a finales de 2018, siendo el mayor porcentaje en la región.

Según la estimación, cerca del $60 \%$ del total de personas migrantes tiene entre 20 y 39 años. Por su parte, los cinco países desde donde proviene la mayoría de ellas son de Venezuela, con 288.233 personas; Perú, con 223.923; Haití, con 179.338; Colombia, con 146.582 personas, y Bolivia, con 107.346 personas. Los venezolanos por primera vez superaron el mayor grupo de migrantes, anteriormente liderado por los peruanos. El segundo grupo que más aumentó en el periodo fue el de los haitianos los cuales alcanzaron un $14,3 \%$ del total de personas extranjeras (INE).

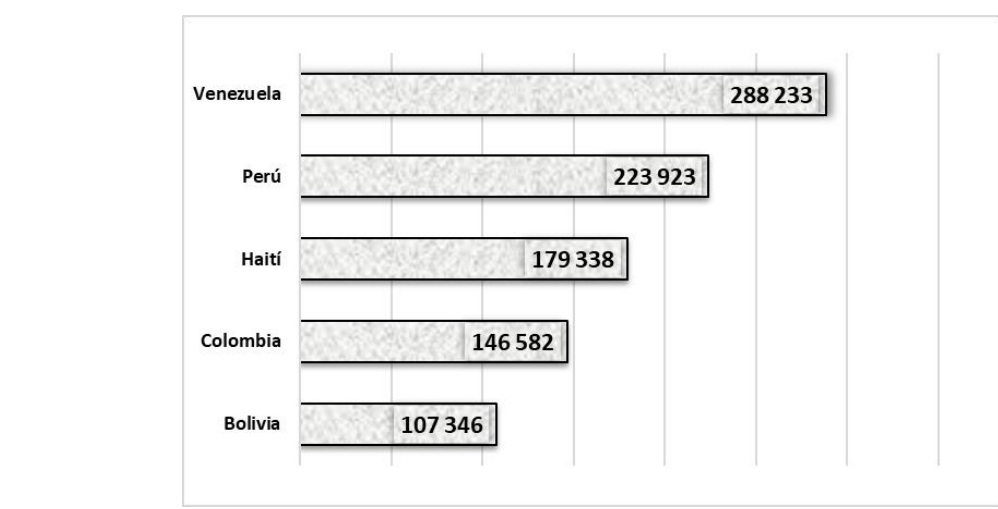

SEQ Gráfico \*ARABIC 2. Gráfico: Número de personas estimadas según país de nacimiento (Fuente: DEM) 
La escolaridad de los migrantes en Chile presenta diferencias importantes a lo típicamente observado en otros países, contrariamente a lo que se puede leer en el estudio de Dustmann (2016), la migración no se ha concentrado en los niveles de educación más bajos, además la mayoría de los migrantes habla español que facilita la integración al mercado laboral. Los chilenos tienen una escolaridad promedio de 11 años, los extranjeros poseen en promedio 12,6 años, mientras que los venezolanos superan todos los grupos con 15,6 años de escolaridad (DEM, 2018). Más del 70\% de los inmigrantes de Venezuela tienen educación terciaria, mientras que para Perú la cifra es solo 19\%. Por otro lado, en lo que respecta a los migrantes con estudios universitarios: solo el 57\% está empleado en ocupaciones de calificación alta comparado con el $87 \%$ de los chilenos universitarios (Banco Central de Chile, 2018).

La migración favorece el país de acogida si la educación y la clase social de los migrantes son más alta, es decir, se produce un efecto positivo sobre el país, aunque puede causar tensiones entre la población privilegiada y los migrantes del nivel socioeconómico alto (Andorka, 1997: 241).

\section{3. ¿Realmente es más fácil buscar un nuevo futuro dentro de la región? ¿Chile facilita la integración laboral de los migrantes?}

Chile es un país estable con una economía sólida, una institucionalidad clara con instituciones democráticas respetadas, por eso es un país atractivo para la población de los países vecinos y actualmente a aquellos más lejanos como Haití, Colombia o Venezuela. Los sueldos son uno de los más altos en América del Sur principalmente, en los puestos que requieren educación terciaria. Los rubros mejor pagados son ingeniería, tecnología, minería (Guía Salarial-Chile, 2019) además el área de salud, principalmente, en el área de medicina, la remuneración y la empleabilidad son altas, debido que esta carrera es la más cara en las universidades chilenas (Ministerio de Educación, Mi futuro).

\subsection{Plazos otorgamiento de permisos de residencia}

Chile ofrece diferentes visas para los extranjeros que quiera vivir en el país. Generalmente, los extranjeros ingresan al país con visa turista y solicitan una visa temporaria en Chile. El gobierno de Sebastián Piñera (quien gobierna por segunda vez desde el 2018 hasta el 2022) quiere acabar con esta práctica y en el futuro solamente en el país de origen se podrá solicitar la visa. "Ha llegado el momento de poner orden en este hogar que compartimos", afirmó el 9 de abril de 2018 el presidente Piñera, fecha en que el gobierno tomó posiciones en cuanto a una política clave en el país: la migración (La Tercera, 06-04-2019). Aunque hubo algunos cambios, el sistema del DEM prácticamente está colapsado, actualmente no se puede ver filas en las calles en el centro de Santiago como antes, pero la espera por obtener una visa es demasiada larga. Además, aumentó la cantidad de los inmigrantes ilegales que ingresan al país como turistas y 
después no solicitan ningún tipo de permiso. El grupo más grande que vive en forma ilegal son los haitianos. Según los datos del DEM cerca de 300 mil inmigrantes ilegales están en el país. El DEM publica los plazos de espera de otorgamiento de permisos de residencia (desde 6,35 meses hasta 24,25 meses), pero en la práctica hay que esperar más tiempo para obtener un permiso de residencia. El problema es más complejo, por ejemplo, para obtener una visa temporaria para profesionales / técnicos, es suficiente el título académico o técnico de la persona y una oferta de trabajo. Según la práctica común y corriente, amigos y parientes ayudan a obtener una oferta de trabajo sin la obligación de contratación. Lamentablemente, con una visa temporaria es muy difícil encontrar un trabajo remunerado, la mayoría de las empresas contratan extranjeros con permanencia definitiva y ésta se otorga después de un año de visa antes indicada.

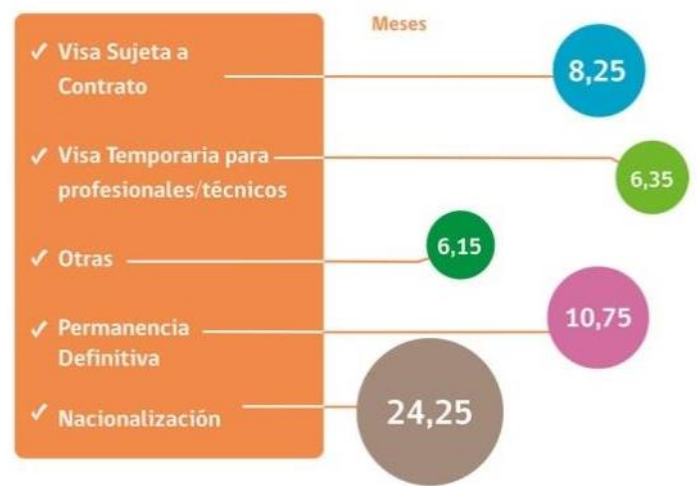

\subsection{Proporción de trabajadores chilenos y extranjeros}

La proporción de los trabajadores chilenos y extranjeros que pueden trabajar en una misma empresa está limitada. Los artículos 19 y 20 del Código del Trabajo establecen que: "el $85 \%$ a lo menos, de los trabajadores que sirvan a un mismo empleador debe ser de nacionalidad chilena [...]" (2002).

Existen excepciones, por ejemplo: si menos de 25 trabajadores trabajan en una empresa, o el extranjero es personal técnico especialista y no existe reemplazo nacional o extranjero cuyo cónyuge, sus hijos sean chilenos etc., o extranjeros residentes por más de cinco años en Chile. Se mencionó anteriormente los extranjeros poseen mayor escolaridad que los chilenos, en especial, los venezolanos, lamentablemente según las tendencias de contratación muchas empresas no tienen vacante para contratar un extranjero, puede ser que la persona sería el candidato perfecto pero la empresa no puede contratarlo debido a la legislación laboral antigua. 
Tendencias actuales y nuevos desafíos de los migrantes en Chile

\subsection{Universidad de Chile - Revalidación de títulos obtenidos en el extranjero}

Se pueden diferenciar tres diferentes trámites: convalidación, reconocimiento y revalidación de títulos obtenidos en el extranjero. Convalidación se refiere a cuando existe un convenio con el país donde fue otorgado el título. Reconocimiento ${ }^{2}$ de título: la Universidad de Chile acepta y certifica que una persona posee un título profesional o un grado académico obtenido en el extranjero, mientras la revalidación: "Corresponde a la certificación de equivalencia entre un título profesional o un grado académico obtenido en el extranjero, con el respectivo título profesional otorgado por la Universidad de Chile u otras instituciones nacionales de educación superior universitaria" (Página de la U. de Chile).

El Ministerio de Educación asignó a la Universidad de Chile la tarea de revalidación y reconocimiento de títulos obtenidos en el extranjero. Por ley es la única institución asignada a esta labor. La revalidación de título es obligatoria en el caso de algunas profesiones, en el área de salud (médico, dentista, etc.), profesores de educación pública y en caso de otras profesiones la revalidación permite libremente ejercer en Chile, es decir en el área privada y pública también.

\subsubsection{El trámite y títulos otorgados}

Desde 2005, la Universidad de Chile publica la lista de las personas que revalidaron los títulos, no existiendo estadísticas, pero la información disponible (título, nombre de la persona, nacionalidad, etc.), permiten recopilar y analizar los datos.

Desde el 2005 hasta el 2018 solamente 4.114 personas revalidaron sus títulos. 20,81\% son de nacionalidad chilena y $80,97 \%$ son extranjeros, según la página de la Universidad de Chile. Con la masiva llegada de los migrantes aumentó el número de los trámites y los venezolanos son el mayor grupo entre los solicitantes. La duración del trámite es de mínimo, unos tres semestres académicos, pero en la práctica puede durar mucho más. El valor de trámite es entre 1000 USD - 1400 USD con un tope de 2.660 USD. Se puede rendir los exámenes únicamente en el idioma español.

Durante la investigación, se entrevistó una chilena que terminó sus estudios en Hungría, en la Academia de Música Franz Liszt, una de las mejores Academias de música en el mundo (University Rankings, 2016). Su título profesional original es Cantante de ópera y profesor de canto, siendo obligada a hacer el trámite debido a que trabaja en una escuela como jefe de coro. La universidad reconoció que sobre $90 \%$ de los ramos son iguales y ella tiene estudios más amplios. Sin importancia de eso, tuvo que hacer el examen final nuevamente, pagar todos los gastos, los ensayos, la sala de concierto, la remuneración del

\footnotetext{
${ }^{2}$ Reconocimiento: El reconocimiento solo procederá cuando el título o grado académico tenga la calidad de título profesional o grado académico de nivel superior en el país de origen, y que el título correspondiente no sea requisito indispensable para el ejercicio profesional en Chile (www.uchile.cl).
} 
profesor guía, el pianista, acompañante etc., además por el valor de su trámite fue un monto que era equivalente a cerca de 2 meses de su remuneración. Debido a que la Universidad de Chile no posee un título similar, el título otorgado por la universidad fue Intérprete musical con mención en canto. Su trámite demoró cerca de 2 años. Es bastante común que los extranjeros que pertenecen a la clase media-alta pagan un magíster en Chile para evitar la revalidación de título y facilitar la contratación en el mercado laboral.

Generalmente, se critica a la Universidad de Chile por el alto costo y la duración del trámite, además por la falta de transparencia. Un extranjero recién llegado al país, sin ingresos fijos, difícilmente puede pagar un trámite que pueda costar hasta 2.660 USD.

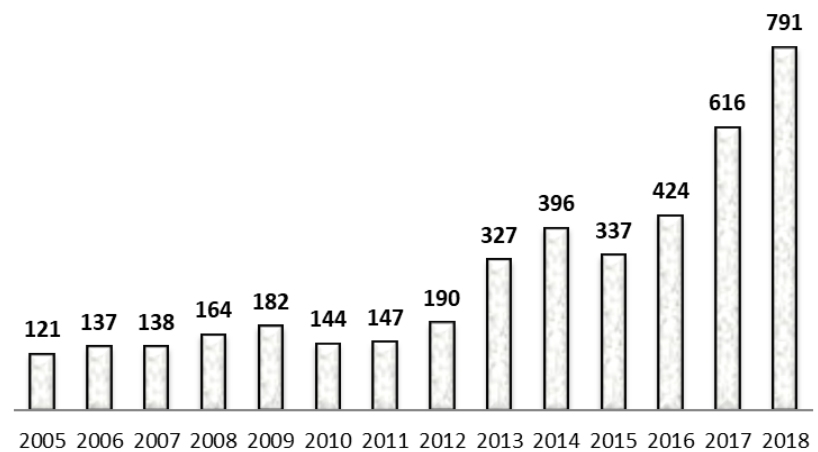

SEQ Gráfico \* ARABIC 4. Gráfico: Revalidación de títulos de la U. de Chile (2005-2018) (Fuente: Elaboración propia base de los datos de la U. de Chile)

\subsubsection{Revalidación de títulos de la Universidad de Chile - Cirujano Dentista / Médico cirujano}

A la continuación, se revisarán dos carreras importantes: el cirujano dentista y el médico cirujano. El DEM publica listas de las visas otorgadas desde el 2005 con información básica (profesión, educación, nacionalidad, tipo de visa etc.) Recopilando la información, el análisis recurre a un tipo de datos que emerge de las diferentes listas, comparando los títulos revalidados por la Universidad de Chile con las visas temporarias otorgadas por el DEM. En el presente estudio solamente los resultados más importantes fueron publicados. Es importante mencionar que existe convenio con Ecuador, Colombia, etc., pero no se permite ejercer la profesión libremente como también requiere algunos exámenes. 
Tendencias actuales y nuevos desafíos de los migrantes en Chile

\subsubsection{Revalidación de títulos de la Universidad de Chile - Cirujano Dentista}

Desde 2005 hasta el 2018 solamente 151 títulos de cirujano dentista fueron reconocidos por la Universidad de Chile, de los cuales, 40 de 151 son de nacionalidad chilena. En contraste, durante estos años, 4.776 dentistas solicitaron visa temporaria. Se puede evidenciar la masiva llegada de los profesionales extranjeros, ya que entre 2017 y 2018, 1.341 dentistas extranjeros solicitaron visa temporaria y cerca de la mitad son venezolanos. Cabe mencionar que actualmente Venezuela no tiene convenio con Chile.

\subsubsection{Revalidación de títulos de la Universidad de Chile -Médico cirujano}

En el análisis se encontró más discrepancia entre la llegada de médicos y la revalidación de títulos por la Universidad de Chile. Entre 2005 y 2018 otorgaron 355 títulos de médico cirujano, de los cuales, 146 de 355 fueron de nacionalidad chilena. Recopilando y comparando los datos del Departamento de Extranjería y Migración durante estos años un total de 12.565 profesionales de médico o médico con especialización solicitó visa temporaria. Los médicos venezolanos lideraron la lista con un total 5.158 visas otorgadas, principalmente entre 2017 y 2018. Como fue mencionado anteriormente, algunos países (Ecuador, Colombia, etc.) tienen convenios si el título o la especialización existen en Chile, pero el convenio no permite trabajar libremente en el país.

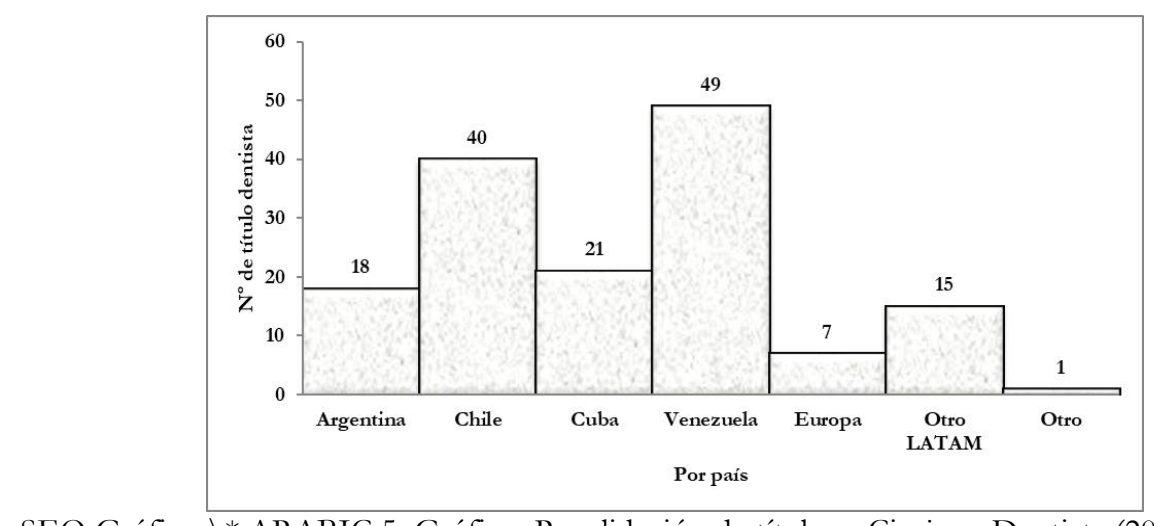

SEQ Gráfico \*ARABIC 5. Gráfico: Revalidación de títulos - Cirujano Dentista (2005-2018)

Total: 151 (Fuente: Elaboración propia base de los datos de la U. de Chile) 


\begin{tabular}{|c|c|c|c|c|c|c|c|c|c|c|c|c|}
\hline Año & $\begin{array}{l}\text { Médico } \\
\text { /Médico } \\
\text { con esp. }\end{array}$ & Argentina & Bolivia & Ecuador & Colombia & Cuba & Perú & Uruguay & Venezuela & Europa & $\begin{array}{l}\text { Otro } \\
\text { LATAM }\end{array}$ & Otro \\
\hline 2018 & 3124 & 54 & 73 & 129 & 221 & 172 & 12 & 7 & 2335 & 20 & 75 & 28 \\
\hline 2017 & 2577 & 83 & 82 & 220 & 246 & 139 & 16 & 8 & 1869 & 27 & 55 & 32 \\
\hline 2011-2016 & 4794 & 321 & 379 & 807 & 1227 & 350 & 58 & 44 & 1127 & 128 & 283 & 94 \\
\hline $2005-2010$ & 2070 & 108 & 208 & 680 & 605 & 69 & 65 & 116 & 27 & 44 & 109 & 41 \\
\hline Total: & 12565 & 564 & 742 & 1836 & 2299 & 730 & 149 & 175 & 5158 & 217 & 502 & 193 \\
\hline
\end{tabular}

Tabla: Visa Temporaria - Profesión: Médico/Médico con especialización (2005-2018)

Total: 12.565 (Fuente: Elaboración propia base de los datos del DEM)

Por supuesto, no todos los médicos con visa temporaria se quedan en Chile, ya que de no poder revalidar los títulos cambian de país o trabajan en otra área.

\subsection{EUNACOM - Examen Único Nacional de Conocimientos de Medicina}

Las dificultades de los profesionales del área de salud no terminan con la Universidad de Chile. Existe otro examen que tienen que rendir si alguien quiere trabajar en el área de la medicina, incluyendo los profesionales con convenio. Por mandato de la Ley 20.261 que entró en vigor en abril del año 2009, los médicos titulados en Chile y en universidades extranjeras deben rendir y haber aprobado el EUNACOM. El examen permite trabajar en el sistema público y es exigido en la mayoría de los centros privados" (Página de la U. de Chile).

El examen es caro (Sección Teórica: 257 USD, Sección Práctica: 600 USD), para el caso de los extranjeros, éstos deben aprobar el examen teórico y práctico; es posible intentarlo hasta dos veces en un año (Página de Eunamed).

Muchos critican el examen, a modo de ejemplo, se obtuvo el siguiente titular, "EUNACOM: 70\% de los médicos que rindió el examen lo había reprobado antes" (La Tercera, 12-07-2017). Los recién titulados pueden rendir mejor, dado que las preguntas son generales en cambio un profesional experimentado que trabaja hace 15-20 años en el área, además con una especialización determinada de estudio y ejercicio, no logra muchas veces aprobar el examen en el primer intento, debido que su conocimiento es más específico. Por supuesto que existen cursos, por lo demás caros para ayudar a la preparación del examen, pudiendo rendirlos únicamente en español.

\subsection{Estudiar Medicina en Chile - Faltan los profesionales}

La página del Ministerio de Educación, “Mi futuro” entrega estadísticas importantes de cada carrera. El número de titulados de la carrera de medicina en 2017 fue de 1.637 (Ministerio de Educación-Mi Futuro, 2017).

La empleabilidad es del 92,4\%, el ingreso bruto mensual: primer año 1.932.470 CLP (2.827 USD), quinto año: 2.866.192 CLP (4.193 USD) son considerablemente altos. 
Duración de la carrera: entre 14-15,3 semestres, el arancel anual es muy diverso, depende de la Universidad, el más barato es de 4.643.000 CLP (6.792 USD,) y el más caro es de 7.684.573 CLP (11.240 USD) (Ministerio de Educación, Mi futuro). Los indicadores en el caso de la carrera de dentista son muy similares, lo cual no será analizado detalladamente. Aunque la empleabilidad es alta y la carrera es bien remunerada, el alto costo del arancel significa una inversión elevada para el estudiante de medicina y su familia. Un estudio encargado por el Ministerio de Salud (Minsal) pronosticó para el año 2030 la falta de 7.200 médicos en la salud pública, 4.772 médicos generales y 2.428 especialistas (Minsal, 2017). Empíricamente los números de titulados de las carreras de medicina en Chile no podrán cubrir esta demanda.

En consecuencia, esta investigación observó que Chile podría resolver el problema de los médicos faltantes. En efecto, comparando con los datos mencionados anteriormente, en el 2018, las visas temporarias otorgadas según profesión "médico /médico con especialización” superaron las 3 mil, mientras el 2017, solo 2.577. Prácticamente, ingresó a Chile el 2/3 de los médicos faltantes, ergo es una pregunta importante: ¿Chile por qué no facilita la revalidación de los títulos en el área de salud? Actualmente el sistema público tiene dificultades grandes, principalmente en provincias ya que están sin médicos ni especialistas.

Los problemas son tan graves que la Contraloría General de la República de Chile en la Región de Arica y Parinacota, en agosto del 2019, dictaminó que se puede contratar a médicos extranjeros sin EUNACOM en salud pública " [...] en situaciones de escasez de médicos y cuando ello sea imprescindible para asegurar la entrega de prestaciones de salud [...]". Los médicos contratados en esta forma tendrían un año de plazo para obtener el examen de EUNACOM (El Mercurio, 23-08-2019).

Según registros de autoridades locales en Arica (extremo norte de Chile con frontera con Perú), cerca de 200 médicos cubren la demanda por salud de poco más de 226 mil habitantes, es decir, un facultativo por cada 1.130 habitantes, mientras que la OCDE recomienda, al menos, un facultativo por cada 322 habitantes (El Mercurio, 23-08-2019).

\subsection{Otros detalles}

No solamente en el área de salud, sino otros profesionales también deben solicitar permisos. Los ingenieros y técnicos graduados en el extranjero, de acuerdo con Ley $\mathrm{N}^{\circ} 12.851$, y en cumplimiento del Art. $6^{\circ}$, deberán solicitar autorización del Colegio de Ingenieros de Chile para su ejercicio. Valor del trámite: 300 USD (Página de Colegio de Ingenieros).

Además, un problema adicional es que Chile no suscribió la Convención sobre la circulación vial de 1968 (Convención de Viena). Solo existe convenio de homologación con Corea del Sur y con España. Todos los extranjeros tienen que hacer el examen teórico, práctico y de salud para obtener una licencia de conducir. Muchas veces es indispensable una licencia chilena por las largas distancias dentro de Santiago. Sin embargo, en el caso de 
un accidente, el seguro no paga si el conductor tiene licencia emitida en el extranjero (Página de Conaset).

El tema de los arriendos es un desafío para cualquier persona en Chile, más aún si es un extranjero el que busca un hogar donde vivir. Para alquilar un departamento, por lo menos se requiere un aval, contrato de trabajo, demostrar situación económica y muchas veces se solicitan varios meses de pago por adelantado. Lo mismo ocurre con la apertura de cuenta corriente porque el banco requiere documentos similares que en el caso anterior.

\section{Conclusiones}

Un mercado laboral atractivo, la seguridad, el bajo nivel de la corrupción y la economía, en conjunto, favorecen la llegada de los migrantes. Actualmente, es bastante común que en el área de servicios trabajen extranjeros, ya que no existen tantas restricciones en esta área, porque las empresas son más pequeñas, facilitando la contratación de los migrantes. Abogados, médicos, dentistas trabajan en los restaurantes como camareros o conductores de Uber, Cabify u otro tipo de taxi. Muchos no pueden revalidar los títulos académicos por falta de ingreso o simplemente no pueden ejercer sus profesiones. En consecuencia, "Chile desaprovecha a inmigrantes con estudios superiores" (La Segunda, 20-06-2018).

Por un lado, existe la demanda de profesionales, especialmente médicos en el área pública, pero los convenios son muy limitados con otros países, además que el trámite es muy largo y costoso. No existe ayuda económica para revalidar los títulos o la llegada de los profesionales que faltan en el país. Todos tienen la misma fila para solicitar una visa o revalidar un título. Prácticamente es más fácil encontrar un trabajo sin educación como empleado de casa particular (es decir, limpiadora) con un contrato que inmediatamente entrega el empleador. La mayoría de los inmigrantes en Chile hablan el mismo idioma, así no se puede comparar la llegada de los migrantes con otros países como EE. UU., en donde muchos profesionales tienen que aprender el idioma local primero para encontrar un trabajo profesional.

Sin duda, si un médico o dentista no puede revalidar su título tiene varias opciones: dejar el país, trabajar en otras áreas, o existe un tercer camino, empezar a trabajar ilegalmente. Cuando alguien busca un dentista de "Tres B", es decir bueno, bonito, barato, muchas veces será un consultorio ilegal. Por supuesto, eso podría ser un tema de una investigación nueva.

Con este estudio se observó además que el sistema actual de Chile, en cuanto a la falta de convenios y el trámite de reconocimiento de títulos, afectan a los chilenos como a los extranjeros. En el siglo XXI, cuando muchos jóvenes quieren estudiar fuera del país de origen, es muy difícil o imposible la inserción a la sociedad chilena, especialmente, si quieren volver a Chile con una pareja extranjera.

Aunque la ley de migración es bastante liberal en Chile, la sociedad le impone más 
restricciones al migrante. Existen políticas, regulaciones y costumbres de los chilenos que no favorecen a la migración, como también falta información de los trámites. Muchos eligen a Chile como destino final porque hablan el mismo idioma y piensan que podrían empezar una nueva vida con más facilidad, pero resulta que es una expectativa falsa. Volviendo a las preguntas iniciales: la respuesta es simple, no es fácil encontrar trabajo y no se facilita la integración laboral de los migrantes en Chile, sin importar que se pudieran obtener muchos profesionales que faltan en el país, evitando los trabajos ilegales u otros inconvenientes que puede causar el sistema actual.

\section{Referencias bibliográficas}

Aguayo, David (2017). EUNACOM: 70\% de los médicos que rindió el examen lo había reprobado antes. La Tercera, 12 de julio.

Andorka, Rudolf (1997). Bevezetés a szociológiába. Budapest: Osiris.

Castellanos, Camilo (2018). Chile desaprovecha a inmigrantes con estudios superiores. La Segunda, 20 de junio.

Chávez, Maximiliano (2019). Salud estima que la brecha de médicos especialistas es de 4.900 facultativos. El Mercurio, 23 de agosto.

Dustmann, Christian-Schönberg - Uta-Stuhler, Jan (2016). The Impact of Immigration: Why Do Studies Reach Such Different Results? Journal of 69 Economic Perspective, 30(4). 31 56.

Gonzalez, Valentina (2019). En 2018, venezolanos lideraron peticiones de extranjeros a Universidad de Chile para revalidar títulos. ElMercurio, 28 de enero.

Navarrete, M. J. - Vedoya, S. (2019). “Ordenar la casa”: análisis del primer año de la política migratoria del gobierno. La Tercera, 06 de abril.

\section{Páginas de Web:}

Banco Central - Chile: Mercado laboral: hechos estilizados e implicancias macroeconómicas diciembre 2018. Asequible en: www.bcentral.cl, fecha de consulta: 05-052019.

Censo 2017: https://www.censo2017.cl/, fecha de consulta: 03-05-2019.

Código del Trabajo - Chile: Art. 19 y 20, 31 de julio de 2002. Asequible en: https://www.leychile.cl/Navegar?idNorma=207436, fecha de consulta: 22-08-2019.

Colegio de Ingenieros de Chile. Asequible en: http://www.ingenieros.cl, fecha de consulta: 03-05-2019.

Comisión Nacional de Seguridad de Tránsito: https://www.conaset.cl/, fecha de 
consulta: 03-05-2019.

Corruption perceptions index 2018. Asequible en: https://www.transparency.org /cpi2018, fecha de consulta: 23-08-2019.

Departamento de Extranjería y Migración. Asequible en: https://www.extranjeria.gob.cl, fecha de consulta: 03-05-2019.

Estimación de Personas Extranjeras Residentes en Chile 2018. Asequible en: https://www.extranjeria.gob.cl/estadisticas-migratorias/, fecha de consulta: 03-05-2019.

Estimación de Personas Extranjeras Residentes en Chile, 31 de diciembre 2018. Asequible en: https://www.ine.cl, fecha de consulta: 06-03-2019.

Fondo Monetario Internacional. Asequible en: https://www.imf.org, fecha de consulta: 05-05-2019.

Guía Salarial - Chile (2019). Asequible en: www.roberthalf.cl, fecha de consulta: 23-082019.

Insight Crime. Chile Profile. InSight Crime - Investigation \& Analysis of Organized Crime. 23 de abril 2018. Asequible en: https://www.insightcrime.org/chile-organizedcrime-news/, fecha de consulta: 10-09-2019.

Instituto Nacional de Estadísticas (INE). Asequible en: www.ine.cl/, fecha de consulta: 05-06-2019.

Médicos del Mundo en Chile. Asequible en: https://eunamed.com/blog/costo-deinscripcion-al-eunacom/, fecha de consulta: 05-06-2019.

Ministerio de Educación, Mi futuro. Asequible en: https://www.mifuturo.cl/, fecha de consulta: 05-06-2019.

Ministerio de Salud (Minsal): Estudio determinación de brecha de médicos generales y especialistas - Informe Final 13-12-2017. Asequible en: https://www.minsal.cl/wpcontent/uploads/2018/03/Estudio-determinaci $\% \mathrm{C} 3 \%$ B3n-de-brechas-m\%C3\%A9 dicos.pdf., fecha de consulta: 06-06-2019.

Revalidación y Reconocimiento de títulos y grados extranjeros en la Universidad de Chile. Asequible en: www.uchile.cl, fecha de consulta: 05-06-2019.

QS World University Rankings by Subject 2016 - Performing Arts. Asequible en: https://www.topuniversities.com/, fecha de consulta: 03-09-2019. 